\title{
Canadian Rheumatology Association Recommendations for the Assessment and Monitoring of Systemic Lupus Erythematosus
}

Stephanie O. Keeling, Zainab Alabdurubalnabi, Antonio Avina-Zubieta, Susan Barr, Louise Bergeron, Sasha Bernatsky, Josiane Bourre-Tessier, Ann Clarke, Alexandra Baril-Dionne, Jan Dutz, Stephanie Ensworth, Aurore Fifi-Mah@, Paul R. Fortin, Dafna D. Gladman, Derek Haaland, John G. Hanly, Linda T. Hiraki, Sara Hussein, Kimberly Legault, Deborah Levy, Lily Lim, Mark Matsos, Emily G. McDonald, Jorge Medina-Rosas, Jordi Pardo Pardi, Christine Peschken, Christian Pineau, Janet Pope, Tamara Rader, Jen Reynolds, Earl Silverman, Konstantinos Tselios, Manon Suitner, Murray Urowitz, Zahi Touma, Evelyne Vinet, and Nancy Santesso

ABSTRACT. Objective. To develop recommendations for the assessment of people with systemic lupus erythematosus (SLE) in Canada.

Methods. Recommendations were developed using the GRADE (Grading of Recommendations Assessment, Development, and Evaluation) approach. The Canadian SLE Working Group (panel of Canadian rheumatologists and a patient representative from Canadian Arthritis Patient Alliance) was created. Questions for recommendation development were identified based on the results of a previous survey of SLE practice patterns of members of the Canadian Rheumatology Association. Systematic literature reviews of randomized trials and observational studies were conducted. Evidence to Decision tables were prepared and presented to the panel at 2 face-to-face meetings and online.

Results. There are 15 recommendations for assessing and monitoring SLE, with varying applicability to adult and pediatric patients. Three recommendations focus on diagnosis, disease activity, and damage assessment, suggesting the use of a validated disease activity score per visit and annual damage score. Strong recommendations were made for cardiovascular risk assessment and measuring anti-Ro and anti-La antibodies in the peripartum period and conditional recommendations for osteoporosis and osteonecrosis. Two conditional recommendations were made for peripartum assessments, 1 for cervical cancer screening and 2 for hepatitis B and C screening. A strong recommendation was made for annual influenza vaccination.

Conclusion. These are considered the first guidelines using the GRADE method for the monitoring of SLE. Existing evidence is largely of low to moderate quality, resulting in more conditional than strong recommendations. Additional rigorous studies and special attention to pediatric SLE populations and patient preferences are needed. (First Release September 1 2018; J Rheumatol 2018;45:1426-39; doi:10.3899/jrheum.171459)

Key Indexing Terms:

SYSTEMIC LUPUS ERYTHEMATOSUS GRADE MONITORING ASSESSMENT

RECOMMENDATIONS COMORBIDITIES

\footnotetext{
From the Division of Rheumatology, Department of Medicine, University of Alberta, Edmonton, Alberta; Division of Rheumatology, Department of Medicine, University of British Columbia, Vancouver, British Columbia; Division of Rheumatology, Department of Medicine, Cumming School of Medicine, University of Calgary, Calgary, Alberta; Canadian Arthritis Patient Alliance; Division of Rheumatology, Department of Medicine, McGill University; Division of Rheumatology, Department of Medicine, Université de Montréal, Montreal; Division of Rheumatology, Department of Medicine, Université Laval, Quebec City, Quebec; Institute of Health Policy, Management and Evaluation, University of Toronto, Toronto; Division of Rheumatology, Department of Medicine, McMaster University, Hamilton, Ontario; Division of Rheumatology, Department of Medicine, Dalhousie University, Halifax, Nova Scotia; Division of Rheumatology, Hospital for Sick Children, Department of Pediatrics, University of
}

Toronto, Toronto, Ontario; Division of Rheumatology, Department of Medicine, University of Manitoba, Winnipeg, Manitoba; General Internal Medicine, McGill University Health Centre, McGill University, Montreal, Quebec; Cochrane Musculoskeletal Group, University of Ottawa, Ottawa; Division of Rheumatology, Department of Medicine, Western University, London, Ontario; Canadian Agency for Drugs and Technologies in Health, Ottawa; Department of Health Research Methods, Evidence and Impact, McMaster University, Hamilton; Centre for Practice-changing Research, Ottawa Hospital Research Institute, Ottawa, Ontario.

These guidelines were supported by a peer-reviewed dissemination event grant from the Canadian Institutes of Health Research, a meeting grant from the Arthritis Society of Canada, and support from the Canadian Rheumatology Association and Lupus Canada. Dr. Clarke holds The Arthritis Society Chair in Rheumatic Diseases at the Cumming School of 
Systemic lupus erythematosus (SLE) is a complex autoimmune disease with multiple phenotypes that can be challenging to diagnose, monitor, and manage over time. In Canada, the prevalence of the disease is 32 to 51 cases per 100,000 individuals ${ }^{1}$. Outcomes are influenced by disease activity and damage, inequities in socioeconomic status, and access to specialist care across Canada ${ }^{1}$. Practice patterns can differ greatly across Canada, as demonstrated in a 63-item questionnaire sent to 175 members of the Canadian Rheumatology Association (CRA) in 2012, the majority being rheumatologists ${ }^{2}$. There was notable heterogeneity among responders for multiple aspects of care, with considerable differences seen in disease activity and damage evalu-

Medicine, University of Calgary. Dr. Fortin holds a tier 1 Canada Research Chair on Systemic Autoimmune Rheumatic Diseases. Drs. Gladman, Touma, and Urowitz were involved in the development of the Systemic Lupus Erythematosus Disease Activity Index (SLEDAI) and the $S L E D A I-2 K$, but do not receive payments from the use of these indices. S.O. Keeling, MD, MSc, FRCP (C), Division of Rheumatology, Department of Medicine, University of Alberta; Z. Alabdurubalnabi, MBBS, ABIM, FRCPC, Division of Rheumatology, Department of Medicine, University of British Columbia; A. Avina-Zubieta, MD, MSc, PhD, FRCPC, Division of Rheumatology, Department of Medicine, University of British Columbia; S. Barr, MD, MSc, FRCPC, Associate Professor, University of Calgary; S. Bernatsky, MD, PhD, FRCPC, Professor of Medicine, McGill University; J. Bourre-Tessier, MD, MSc, FRCPC, Associate Clinical Professor, Université de Montréal; A. Clarke, MD, MSc, FRCPC, Professor of Medicine, Cumming School of Medicine, University of Calgary; A. Baril-Dionne, MD, Internal Medicine Resident, Université de Montréal; J. Dutz, MD, FRCPC, Professor of Medicine, University of British Columbia; S. Ensworth, MD FRCPC, Clinical Assistant Professor, University of British Columbia; A. Fifi-Mah, MD, FRCPC, Clinical Assistant Professor, University of Calgary; P.R. Fortin, MD, FRCPC, Professor of Medicine, Université Laval; D.D. Gladman, MD, FRCPC, Professor of Medicine, University of Toronto; D. Haaland, MD, MSc, FRCPC, Assistant Clinical Professor, McMaster University; J.G. Hanly, $M D, F R C P C$, Professor of Medicine, Dalhousie University; L.T. Hiraki, $M D, F R C P C, S c D$, Assistant Professor, University of Toronto; S. Hussein, $M D$, Internal Medicine Resident, Université de Montréal; K. Legault, MD, MSc, FRCPC, Assistant Professor, McMaster University; D. Levy, MD, MSc, FRCPC, Associate Professor, University of Toronto; L. Lim, MBBS, MRCPCH, FRCPC, PhD, Assistant Professor, University of Manitoba; M. Matsos, MD, FRCPC, Associate Professor, McMaster University; E.G. McDonald, MD, FRCPC, Assistant Professor, McGill University; J. Pardo Pardi, Co-Managing Editor, Cochrane Musculoskeletal Group, Centre for Practice-changing Research, Ottawa Hospital Research Institute; C. Peschken, MD, FRCPC, Associate Professor, University of Manitoba; C. Pineau, MD, FRPCP, Associate Professor, McGill University; J. Pope, MD, FRCP $(C)$, Professor of Medicine, Western University; T. Rader, MLIS, Canadian Agency for Drugs and Technologies in Health; J. Reynolds, MD, FRCPC, Clinical Assistant Professor, University of British Columbia; E. Silverman, MD, FRCPC, Professor of Pediatrics, University of Toronto; K. Tselios, MD, PhD, Clinical Research Fellow, University of Toronto; M. Suitner, MD, Internal Medicine Resident, Université de Montréal; $M$. Urowitz, MD, FRCPC, Professor of Medicine, University of Toronto; Z. Touma, MD, PhD, Assistant Professor, University of Toronto; E. Vinet, MD, PhD, Assistant Professor, McGill University; N. Santesso, RD, PhD, Assistant Professor, McMaster University. Louise Bergeron and Dr. Jorge Medina-Rosas died before the manuscript was completed.

Address correspondence to Dr. S.O. Keeling, 8-129 Clinical Sciences Building, University of Alberta, Edmonton, Alberta T6G 2 G3, Canada. E-mail: Stephanie.keeling@ualberta.ca

Full Release Article. For details see Reprints and Permissions at jrheum.org Accepted for publication July 13, 2018. ations, cardiovascular (CV) risk, and peripartum assessments in people with SLE.

Previous recommendation efforts in SLE have acknowledged the utility of evidence-based guidance to identify minimally important monitoring for patients with SLE, to reduce variability in clinical heterogeneity ${ }^{3}$. In addition to organizing monitoring of patients with SLE in clinic, recommendations are also important to provide guidance on optimal treatment of these patients, given the existing and emerging therapeutics in SLE. Internationally, existing recommendations for management of patients with SLE include the European League Against Rheumatism recommendations for monitoring patients with $\mathrm{SLE}^{3}$, the American College of Rheumatology (ACR) guidelines for screening, treatment, and management of lupus nephritis ${ }^{4}$, and the British Society of Rheumatology guidelines for the management of $\mathrm{SLE}^{5}$. These organizations used comprehensive methodologies including systematic literature reviews, expert panels, face-to-face meetings, and voting rounds to develop their respective recommendations. Similar methodology was recently used to develop quality indicators in SLE, an important complement to recommendations ${ }^{6}$. However, limitations of these guidelines include the lack of incorporation of patient preferences, and other important issues such as acceptability, feasibility, and equity in the guideline development process.

The Grading of Recommendations Assessment, Development, and Evaluation (GRADE) working group has developed one of the leading frameworks and methodologies for clinical practice guideline/recommendation development since its beginnings in $2000^{7-13}$. Over 100 organizations including the ACR and CRA have adopted the GRADE framework ${ }^{14}$. This methodology includes a careful assessment of the evidence, and provides a rating of the level of evidence and "strength" of a recommendation (e.g., strong or conditional). This approach ensures rigorously developed recommendations based on the quality of evidence, patient preferences and values, and feasibility, acceptability, cost, and equity considerations.

We aimed to develop recommendations for the assessment and monitoring of SLE to provide guidance for SLE healthcare providers, in particular Canadian rheumatologists. These recommendations may also prove useful for other adult and pediatric subspecialists involved in SLE care in addition to other specialty-specific guidelines ${ }^{4}$. These recommendations are the first to use the GRADE methodology for SLE, to our knowledge, and are endorsed by the CRA, the Canadian Network for Improved Outcomes in SLE, Lupus Canada, and SLE centers across Canada.

\section{MATERIALS AND METHODS}

The Canadian SLE Working Group, composed of 23 adult rheumatologists, 4 pediatric rheumatologists, 1 immunologist, 4 general internal medicine and rheumatology trainees, and 1 patient representative from the Canadian Alliance for Patients with Arthritis was created to develop recommendations

Personal non-commercial use only. The Journal of Rheumatology Copyright $\subset$ $(2018$. All rights reserved. 
for the assessment and monitoring of SLE. The International Committee of Medical Journal Editors conflict of interest form was completed and reviewed prior to the face-to-face meetings for recommendation development. GRADE methodology and systematic literature review development were guided by a GRADE representative (NS) and Cochrane Musculoskeletal Librarians (JPP/TR).

Formulating questions and determining outcomes - the Practice Pattern Survey. In February 2013, the Canadian SLE Working Group, synonymous with the guideline panel, met for the first time to evaluate the results of the SLE practice pattern survey sent to 175 members of the CRA in the previous year. The panel developed a series of questions regarding assessment and monitoring of SLE from these results and prioritized and ranked them during and after the meeting through online Survey Monkey voting. A series of 8 different areas concerning SLE clinical care were determined as areas from which GRADE-based recommendations would be developed, informed in part by the significant heterogeneity in practice patterns, including (1) healthcare provider for SLE; (2) disease activity and damage; (3) osteoporosis; (4) osteonecrosis; (5) CV risk assessment; (6) peripartum assessment; (7) cervical cancer screening; and (8) infection screening and prevention (hepatitis B, C, and influenza). Other areas were important but deemed as lower priorities than the listed topics: renal biopsies, vaccination for pneumonia, varicella zoster, hepatitis B and human papilloma virus, and other malignancy screening. These will be considered in future iterations of the recommendations, depending upon feasibility. Detailed assessments of lupus nephritis and thrombotic risk related to antiphospholipid antibody syndrome (APS) in SLE and pregnancy were not included.

Systematic review of the evidence. Eight groups composed of members of the Canadian SLE Working Group were organized to conduct systematic literature reviews to develop Evidence to Decision tables (ETD) under the guidance of the Cochrane Musculoskeletal Group (JP, TR) and GRADE (NS). Webinar and teleconferences were conducted to formulate the PICO (Population, Intervention, Comparison, Outcome) search strategy. Each group searched Medline, Embase, and PubMed from 1946 (beginning of Medline) to at least February 2016 (immediately preceding the first guideline voting meeting) or more recently (as indicated in individual ETD tables) for randomized and nonrandomized studies for assessment and monitoring, and for studies reporting prevalence of comorbidities and infection-related risks. References were also hand-searched for relevant articles. General exclusions included non-English articles, case series, and case reports. The Newcastle-Ottawa scale for observational studies was used for the majority of studies because they were largely observational ${ }^{15}$. This method awards a maximum of 1 star (high quality) in 3 domains including selection of the study groups, the comparability of the groups, and the ascertainment of outcome of interest if criteria are met. The higher number of stars represents a higher quality of study. Quality in Prognosis Studies was used by some groups for assessing prognosis studies ${ }^{16}$. This is a risk-of-bias tool for assessing 6 common domains of prognosis studies: study population, attrition, outcomes, prognostic factors, confounders, and statistical analysis. The Cochrane Risk of Bias tool was used to evaluate the quality of randomized trials ${ }^{17}$. Metaanalyses were performed when data from the studies was in a form that could be pooled. Specific details for each systematic literature review are available in the respective published manuscripts. The evidence was assessed using the GRADE approach and presented in GRADE evidence tables and ETD tables ${ }^{18,19}$ (Supplementary Data, available with the online version of this article).

Development of recommendations. A preliminary meeting was held in Toronto, Canada, on September 18-19, 2015, for the panel to discuss GRADE methodology and review the status of the ETD. A final meeting was held on February 16-17, 2016, to review the tables and develop recommendations. This meeting, chaired by SK and NS, included a quorum of the working group. Several members participated remotely through teleconferencing. ETD were presented and discussed and voted upon by the panel. Areas requiring clarification were identified for post-meeting discussion. Recommendations for influenza vaccination and hepatitis screening were developed post-meeting with review of the ETD and voting conducted through the GRADEpro software (www.gradepro.org). Because of time constraints, screening of varicella zoster and hepatitis $\mathrm{B} / \mathrm{C}$ virus titers as well as hepatitis B vaccination were not pursued. Recommendations were finalized by the panel and presented at the CRA 2017 Annual Scientific Meeting in February 2017 in Ottawa, Canada. The recommendations are presented as "strong" or "conditional" (Table 1) with implications of recommendation rating outlined in Table 2. Best-practice statements were also developed for an intervention when the benefits were large and unequivocal, the rationale clear, and GRADE was not applied ${ }^{20,21}$. Ethics approval was not required in accordance with the policies of our institutions.

\section{RESULTS}

We developed recommendations for the assessment and monitoring of Canadian patients with SLE based on the observations of heterogeneity in a practice pattern survey of Canadian rheumatologists (Table 3). These recommendations focus on pertinent clinical assessments of patients with SLE that integrate best clinical practice with evidence-based strategies for optimal assessments.

\section{General Assessment Recommendations}

Best clinical practice statement (general assessment). Best clinical practice dictates that all adult and pediatric patients with SLE have a complete history, physical, and laboratory evaluation at baseline and during each followup visit. Careful interpretation of the clinical and laboratory findings is required to ensure proper attribution of the signs, symptoms, and investigation results toward SLE or other comorbid conditions.

Remarks: Best clinical practice includes a complete history and physical examination at baseline, with laboratory monitoring possibly including but not limited to complete blood count (CBC), liver enzymes, creatine kinase, creatinine and estimated glomerular filtration rate (eGFR), urine routine/microscopic (urinalysis), urine protein-creatinine ratio, C-reactive protein (CRP), erythrocyte sedimentation rate (ESR), complements $(\mathrm{C} 3, \mathrm{C} 4)$, anti-dsDNA, antinuclear antibodies, antibodies to extractable nuclear antigens, antiphospholipid antibodies (aPL), lupus anticoagulant

Table 1. Quality of evidence: Certainty in effect ${ }^{8}$.

Rating of Evidence Definition
Quality

High We are very confident that the true effect lies close to that of the estimate of the effect.

Moderate We are moderately confident in the effect estimate. The true effect is likely to be close to the estimate of the effect, but there is a possibility that it is substantially different.

Low $\quad$ Our confidence in the effect estimate is limited. The true effect may be substantially different from the estimate of the effect.

Very low We have very little confidence in the effect estimate. The true effect is likely to be substantially different from the estimate of effect. 
Table 2. Implications of strong and conditional recommendations using the GRADE approach ${ }^{21}$.

\begin{tabular}{lll}
\hline Implications & Strong Recommendation & Conditional Recommendation \\
\hline For patients & $\begin{array}{l}\text { Most individuals in this situation would want the recommended } \\
\text { course of action, and only a small proportion would not. } \\
\text { Formal decision aids are not likely to be needed to help } \\
\text { individuals make decisions consistent with their values } \\
\text { and preferences. } \\
\text { Most individuals should receive the recommended course of action. } \\
\text { Adherence to this recommendation according to the guidelines } \\
\text { could be used as a quality criterion or performance indicator. }\end{array}$ & $\begin{array}{l}\text { The majority of individuals in this situation would } \\
\text { want the suggested course of action, but many } \\
\text { would not. }\end{array}$ \\
$\begin{array}{ll}\text { For clinicians } \\
\text { be appropriate for each individual and that clinicians } \\
\text { must help each individual arrive at a management } \\
\text { decision consistent with the individual's values and } \\
\text { preferences. Decision aids may be useful to help } \\
\text { individuals make decisions consistent with their } \\
\text { values and preferences. } \\
\text { Policy making will require substantial debate and the } \\
\text { involvement of many people. }\end{array}$ & The recommendation can be adopted as policy in most situations. \\
\hline
\end{tabular}

GRADE: Grading of Recommendations Assessment, Development, and Evaluation working group.

(LAC), anticardiolipin (aCL), anti- $\beta_{2}$-glycoprotein I (anti- $\beta_{2}-\mathrm{GPI}$ ), and lipid profile. Followup laboratory monitoring will depend on the patient's clinical status and may include CBC, eGFR, urinalysis, urine protein-creatinine ratio, CRP, and/or ESR, C3, C4, and anti-dsDNA antibodies.

Justification: There is no current evidence that compares outcomes when specific tests are performed or not performed at baseline or at followup. This best-practice statement is therefore based on the utility of results to inform subsequent care of the patient with SLE. For some tests, there is evidence that the results may also be predictive of other health risks (see the following recommendations).

SLE healthcare provider (Supplementary Data 1, Lupus Healthcare Provider, available with the online version of this article). (1) We recommend that all adult patients suspected of SLE be referred to an SLE specialist, most often a rheumatologist, to confirm diagnosis and be involved in ongoing care (strong recommendation; moderate-quality evidence). Remarks: This recommendation considers that the primary care physician continues to provide overall care to the patient, including monitoring for and managing comorbidities.

Justification: There is moderate-quality evidence that care provided by caregivers with more experience treating SLE (typically the rheumatologist) likely results in accurate diagnosis of SLE when compared with primary care physicians $^{22,23,24}$. There is also moderate-quality evidence for lower mortality and hospitalization when an SLE specialist is involved in the care of people with $\operatorname{SLE}^{25,26,27,28}$. Moreover, recommendations of this committee for monitoring patients with SLE include periodic assessment with disease activity and damage indices that are beyond the scope of most primary care physicians, and require experience to attribute results to SLE or other comorbid conditions (see Disease activity and Damage recommendations \#2 and \#3). Regardless of care provider, engaging the patient as a member of the care team and participating in shared care models is important.

Disease activity (Supplementary Data 2, Disease Activity, available with the online version of this article). (2) For adult and pediatric patients with SLE, we suggest assessing disease activity with a validated instrument of disease activity during baseline and followup visits (conditional recommendation, low-quality evidence).

Remarks: This recommendation does not specify what validated instrument should be used; however, examples of validated instruments that may be used include the following: SLE Disease Activity Index-2K $\mathrm{K}^{5,29}$, British Isles Lupus Assessment Group score ${ }^{5,30}$, SLE Activity Measure ${ }^{5,31}$, and others. Several factors influence the choice of a particular instrument including physician preference and expertise, cost, time burdens, and applicability to pediatric populations. All variables in each instrument are derived from a complete history and physical and laboratory examination, which is good clinical practice.

Justification: The evidence showing that measuring disease activity with validated instruments results in better outcomes compared to best clinical practice (complete history, physical and laboratory examinations) is low to moderate quality; however, studies show that higher disease activity is associated with small increases in the risk of death and disease damage, and adverse CV outcomes ${ }^{32}$ (see Cardiovascular Recommendations). The panel agreed that using validated instruments may organize the clinical encounter with the patient, incur negligible costs, and be acceptable to patients; however, the use of disease activity instruments may lead to variable equity in sites owing to inaccessibility to an experienced user of the tool. The panel expressed concern that disease activity measurement with validated tools could still lead to overmeasurement and therefore overtreatment of patients with low disease activity.

Personal non-commercial use only. The Journal of Rheumatology Copyright @ 2018 . All rights reserved. 
General assessment

Best clinical practice dictates that all adult and pediatric patients with SLE have a complete history, physical, and laboratory evaluation at baseline and during each followup visit. Careful interpretation of the clinical and laboratory findings is required to ensure proper attribution of the signs, symptoms, and investigation results towards SLE or other comorbid conditions.

SLE healthcare provider (1) We recommend that all adult patients suspected of SLE be referred to an SLE specialist, most often a rheumatologist, to confirm diagnosis and be involved in ongoing care.

Disease activity (2) For adult and pediatric patients with SLE, we suggest assessing disease activity with a validated instrument of disease activity during baseline and followup visits.

Damage

(3) For adult and pediatric patients with SLE, we suggest assessing disease damage annually with a validated measure.

Cardiovascular $(\mathrm{CV})$ risk assessment

Best practice dictates that a CV risk assessment be performed in adult patients upon diagnosis of SLE.

$\mathrm{CV}$ risk assessment

(4) For adults with SLE, we recommend that indicators of obesity, smoking status, arterial hypertension, diabetes, and dyslipidemia be measured upon diagnosis of SLE, and be reassessed periodically according to current recommendations in the general population and be used to inform the $\mathrm{CV}$ risk assessment.

(5) For adults with SLE, we suggest that carotid ultrasonography not be a part of the $\mathrm{CV}$ risk assessment, except in highly selected cases where expertise is available.

Osteoporosis Osteoporosis

(6) For all adult patients with SLE, we suggest assessing for risk of osteoporosis and fractures every 1 to 3 years using a detailed history and focused physical examination, and measuring bone mineral density in patients with other risk factors according to recommendations in the general population.

(7) For all adults with SLE, we suggest screening for 25-hydroxy vitamin D as part of the assessment for risk of osteoporosis and fractures.

Strong recommendation, moderate-quality evidence

Conditional recommendation, low-quality evidence

Conditional recommendation, low-quality evidence

Strong recommendation, high-quality evidence

Conditional recommendation, low-quality evidence

Conditional recommendation, low-quality evidence

Conditional recommendation, low-quality evidence

Osteonecrosis

Best clinical practice dictates that adult and pediatric patients with SLE, in particular patients who have a history of glucocorticoid exposure, receive information about the symptoms of osteonecrosis, including progressive or sudden deep joint pain that is worse with weight-bearing.

Osteonecrosis

(8) For adult and pediatric patients with SLE who do not have clinical symptoms suggestive of osteonecrosis, we suggest not screening for or Conditional recommendation, low-quality performing investigations for osteonecrosis. For patients who have suspected clinical symptoms of osteonecrosis, we suggest radiographs as the initial imaging modality rather than MRI or bone scan with SPECT, according to recommendations in the general population.

Peripartum assessment

Best practice dictates that all women living with SLE who are planning a pregnancy or who become pregnant should have their individual situations discussed with experts in the area, with referral to an SLE care provider and obstetrical care providers, and an overall plan should be made for their pregnancy care. Best practice dictates that for women with SLE, a complete history, physical, and laboratory evaluation be provided immediately prior to pregnancy and each trimester of pregnancy, and when flare is suspected during pregnancy. Laboratory evaluation should include antiphospholipid antibodies (see Best Clinical Practice General Assessment), with further testing depending on results.

Peripartum assessments $\quad$ (9) For women with SLE, we recommend that anti-Ro and anti-La antibodies be measured prior to pregnancy or during the first trimester. (10) For pregnant women with SLE, we suggest that uterine and umbilical Doppler studies be performed in the second or third trimester, or at the time of a suspected flare.

(11) For women with prior or active lupus nephritis who are pregnant, we suggest measuring serum creatinine and urine protein to creatinine ratio every 4-6 weeks, or more frequently if clinically indicated. We suggest blood pressure and urinalysis be measured prior to pregnancy and every 4-6 weeks until 28 weeks, every 1-2 weeks until 36 weeks, and then weekly until delivery.

Cervical cancer screening

Cervical cancer

screening
(12) For all female adult patients with SLE who are or have been sexually active, regardless of sexual orientation, we suggest annual cervical cancer screening rather than screening every 3 years, at least up to the age of 69 .
Strong recommendation, low-quality evidence

Conditional recommendation, low-quality evidence

Conditional recommendation, low-quality evidence

Conditional recommendation, low-quality evidence 
Infection screening and vaccination

Infection screening/ (13) We recommend that adults and children with SLE receive an vaccination

(13) We recommend that adults and children with SLE receive an
annual inactivated influenza vaccination in a single dose.
(14) For adult and pediatric patients with a diagnosis of SLE and

high-risk behaviors for hepatitis B virus acquisition, we recommend

screening for $\mathrm{HbsAg}$ and repeating according to recommendations for

the general population. For patients being considered for

immunomodulatory therapy, we suggest screening before starting treatment.

(15) For adults and pediatric patients with a diagnosis of SLE and high-risk

behaviors for hepatitis $\mathrm{C}$ virus (HCV) acquisition, we recommend screening

for $\mathrm{HCV}$ and repeating according to recommendations in the general

population. For all other adult and pediatric patients with a diagnosis of SLE,

we suggest screening for $\mathrm{HCV}$ and repeating according to recommendations

in the general population.
Strong recommendation, moderate-quality evidence

Conditional recommendation, low-quality evidence

Conditional recommendation, low-quality evidence

MRI: magnetic resonance imaging; SPECT: single photon emission-computed tomography.

The recommendation considers resource requirements to be negligible but recognizes variability depending on what score is used.

Damage (Supplementary Data 3, Damage, available with the online version of this article). (3) For adult and pediatric patients with SLE, we suggest assessing disease damage annually with a validated measure (conditional recommendation; low-quality evidence).

Remarks: This recommendation does not specify what disease damage tool to use; however, the Systemic Lupus International Collaborating Clinics/ACR damage index $(\mathrm{SDI})^{33}$ is the only validated physician-completed measure to evaluate damage. The components of damage are derived from the full patient assessment as per best clinical practice including a complete history, and physical and laboratory examinations. The SDI measures damage from any cause, including but not limited to SLE since the diagnosis of $\mathrm{SLE}^{33}$.

Justification: The evidence showing better outcomes for patients with SLE (beyond the full history, physical and laboratory examinations) by measuring damage with a validated instrument is low to moderate quality; however, studies show that early and late damage from SLE is associated with small to moderate increases in mortality and future damage, which may lead to reduced function and quality of life ${ }^{32}$. Higher disease damage is also likely associated with small increases in CV outcomes ${ }^{34}$. The panel acknowledged that validated damage instruments provided extra value by allowing for quantification of disease damage in a standardized way that can be followed over time. The panel did not identify any harm from using a validated tool or significant resource requirements but could not demonstrate a cost savings. The panel agreed that the tool would be feasible to implement because it is performed infrequently (e.g., annually) over time and adds minimal time burden. The panel agreed that use of a validated instrument might be more acceptable to patients than rheumatologists because of possible unfamiliarity performing disease damage measures by rheumatologists and their perception of increased time required for completion.

\section{Cardiovascular Risk Assessment Recommendations}

Best clinical practice statement (CV risk). Best practice dictates that a CV risk assessment be performed in adult patients upon diagnosis of SLE (Supplementary Data 4, Cardiovascular Risk ETD, available with the online version of this article).

Remarks: The assessment includes documentation of characteristics of patients that are nonmodifiable but predictive of $\mathrm{CV}$ events, such as age, sex, and family history of premature coronary artery disease in the general population (males $<55$ yrs old, females $<65$ yrs old $)^{35}$. These characteristics and assessments in the complete history, physical, and SLE-related laboratory examinations provided at baseline and followup visits can inform the overall assessment of CV risk (see Best Practice Statement for General Assessment).

Justification: There is high-quality evidence that increasing age is likely associated with an increased risk of CV events, in particular for postmenopausal women and patients with SLE over the age of 48 years $^{34}$. Being male and having a positive family history for premature coronary artery disease are also likely associated with a large increase in risk ${ }^{34,35}$. Regarding the other tests included in the general assessment, studies show that tests confirming renal (including serum creatinine levels) or neuropsychiatric involvement may be associated with moderately increased risks of $\mathrm{CV}$ events and surrogate outcomes ${ }^{34}$. In addition, aPL, particularly aCL and anti- $\mathrm{B}_{2}$-GPI antibodies as well as LAC are likely associated with $\mathrm{CV}$ and cerebrovascular outcomes ${ }^{34}$. CRP, in particular elevated high-sensitivity CRP, may also be associated with moderate risk of $\mathrm{CV}$ events and surrogate outcomes and infection ${ }^{34}$. Disease activity and damage scores have also shown a positive association with $\mathrm{CV}$ risk ${ }^{34}$.

Traditional CV risk factors. (4) For adults, we recommend Personal non-commercial use only. The Journal of Rheumatology Copyright () 2018. All rights reserved. 
that indicators of obesity, smoking status, arterial hypertension, diabetes, and dyslipidemia be measured upon diagnosis of SLE, and be reassessed periodically according to current recommendations in the general population and be used to inform the $\mathrm{CV}$ risk assessment (strong recommendation, high-quality evidence).

Remarks: Indicators for obesity include body weight and body mass index. The 2006 Canadian Obesity Network clinical practice guidelines provide recommendations for action after assessment (www.cmaj.ca/content/suppl/2007/ 09/04/176.8.S1.DC1/obesity-lau-onlineNEW.pdf). For smoking, the Canadian Smoking Cessation clinical practice guidelines (www.strokebestpractices.ca/wp-content/uploads/2012/04/ CAN-ADAPTT2.pdf) provide recommendations for action after assessment. The 2015 Canadian Hypertension Education Program (guidelines.hypertension.ca/diagnosis-assessment) provides information about the measurement and documentation of blood pressure, reassessment and treatment. Initial diabetes screening includes fasting plasma glucose and/or glycosylated hemoglobin. The Canadian Diabetes Association 2013 clinical practice guidelines for patients with $\geq 1$ risk factor provide additional information about reassessment, monitoring, and treatment ${ }^{36}$. Initial dyslipidemia risk evaluation includes basic lipid profile assessment [total cholesterol (TC), triglycerides (TG), high-density lipoproteins (HDL), low-density lipoproteins]. In the case of normal values, periodic reassessment is based on the effect of alterations of disease activity and administered drugs, such as corticosteroids, on lipid metabolism. The 2016 Canadian Cardiovascular Society guidelines provide information for monitoring and treating people at intermediate to high risk of $\mathrm{CV}$ outcomes ${ }^{35}$.

Justification: This recommendation is based on general population recommendations for screening and monitoring of indicators of $\mathrm{CV}$ risk as well as evidence about the potential for increased risk of CV events in patients with $\mathrm{SLE}^{34,35}$. Studies show that there is likely a large increased risk of $\mathrm{CV}$ events with smoking 34,35 . Obesity may be associated with small to large increases in surrogate outcomes related to $\mathrm{CV}$ disease ${ }^{34,35}$. Hypertension is likely related to a small to large increased risk of $\mathrm{CV}$ events, and surrogate outcomes. Increased insulin and glucose may be associated with increased risk of surrogate outcomes, and diabetes is associated with moderate to large increased risk of CV events and surrogate outcomes ${ }^{34,35}$. There is likely a large increased risk of CV events when TC is elevated, and when HDL is low $^{34,35}$. TG may be associated with small to large increased risk of $\mathrm{CV}$ events and surrogate outcomes $^{34,35}$. Because these indicators are available and assessed in primary level care, the panel agreed that the tests were feasible, acceptable, equitable, and would not incur additional costs to perform.

Carotid ultrasonography (US). (5) For adults with SLE, we suggest that carotid US not be a part of the CV risk assessment, except in highly selected cases where expertise is available (conditional recommendation; moderate-quality evidence).

Remarks: The test may provide additional information to risk-stratify patients, particularly in the context of cerebrovascular events (secondary prevention) and those with 1 or more traditional $\mathrm{CV}$ risk factors. This test is characterized by a high rate of false-positive results, hence requiring special expertise.

Justification: There is no evidence comparing the risk of CV outcomes in patients with SLE when results from a carotid US are used or not used in the US risk assessment. Two studies show that carotid artery intima-media thickness is likely associated with a small increased risk of CV events, and total plaque area of the carotid artery is likely associated with a large increased risk ${ }^{34}$. Carotid US requires substantial resources, is not feasible in some institutions, and will probably reduce equity. Therefore, it is suggested that carotid US be reserved only for selected cases and not all adults with SLE.

\section{Osteoporosis Recommendations}

Osteoporosis (Supplementary Data 5, Osteoporosis, available with the online version of this article). (6) For all adult patients with SLE, we suggest assessing for risk of osteoporosis and fractures every 1 to 3 years using a detailed history and focused physical examination, and measuring bone mineral density (BMD) in patients with other risk factors according to recommendations in the general population (conditional recommendation; low-quality evidence).

Remarks: The assessment can be based on the Fracture Risk Assessment Tool (FRAX; in individuals older than $50 \mathrm{yrs}$ ), which includes factors such as age, sex, weight, previous history of fracture, smoking and alcohol habits, use of high-risk medications including glucocorticoids, and height measurement (www.sheffield.ac.uk/FRAX) ${ }^{37}$. BMD is especially measured in individuals with a history of fragility fractures, who have longterm glucocorticoid therapy ( $>3$ mos), who use other high-risk medications, or who have hypogonadism or premature menopause. This recommendation does not apply to the pediatric SLE population, where osteoporosis is defined by fractures and not BMD (www.iscd.org/official-positions/2013-iscd-officialpositions-pediatric).

Justification: Current guidelines in Canada recommend assessing risk of osteoporosis in individuals over age 50 every 1 to 3 years $^{38}$. There is no evidence comparing outcomes related to osteoporosis in patients with SLE who had or did not have an assessment before age 50. However, studies show that the prevalence of osteoporosis or osteopenia and incidence of fractures may be higher in adult women with SLE (mean age $45 \mathrm{yrs})^{39,40,41,42,43,44}$. The panel

Personal non-commercial use only. The Journal of Rheumatology Copyright @ 2018 . All rights reserved. 
agreed that the FRAX tool is likely applicable to patients with SLE, and that measuring BMD can be consistent with recommendations for the general population ${ }^{38}$. There was some concern that facilities to measure BMD may not be available in northern or rural communities, and there may be additional burden to individuals if there is not a nearby bone densitometer.

(7) For all adults with SLE, we suggest screening for 25(OH)D (25-hydroxy vitamin D) as part of the assessment for risk of osteoporosis and fractures (conditional recommendation; low-quality evidence).

Remarks: This recommendation excludes pediatric patients with SLE as the evidence for $25(\mathrm{OH}) \mathrm{D}$ screening was limited to adult patients with SLE.

Justification: There is no evidence comparing assessment of vitamin $\mathrm{D}$ to no assessment and the effects on bone outcomes. However, studies show that vitamin D deficiency may be more prevalent in patients with SLE, in particular, patients with renal failure, glucocorticoid treatment, or photosensitivity $45,46,47,48$. The panel agreed that there would be negligible costs when screening for vitamin $\mathrm{D}$, and it would be acceptable, equitable, and feasible. Followup on the status of vitamin D levels might be considered after a period of treatment.

\section{Osteonecrosis Recommendations}

Best clinical practice statement (Osteonecrosis; Supplementary Data 6, Asymptomatic Osteonecrosis, and 7, Symptomatic Osteonecrosis, available with the online version of this article). Best clinical practice dictates that adult and pediatric patients with SLE, in particular patients who had a history of glucocorticoid exposure, receive information about the symptoms of osteonecrosis, including progressive or sudden deep joint pain that is worse with weight-bearing.

Osteonecrosis (asymptomatic and symptomatic). (8) For adult and pediatric patients with SLE who do not have clinical symptoms suggestive of osteonecrosis, we suggest not screening for or performing investigations for osteonecrosis. For patients who have suspected clinical symptoms of osteonecrosis, we suggest radiographs as the initial imaging modality rather than magnetic resonance imaging (MRI) or bone scan with single photon emission-computed tomography (SPECT) according to recommendations in the general population (conditional recommendation, low-quality evidence).

Justification: There is no evidence comparing outcomes of patients with SLE with or without suspicion of osteonecrosis who are screened or not screened with radiographs. Studies show that the prevalence of osteonecrosis and asymptomatic osteonecrosis may be higher in patients with $\mathrm{SLE}^{49,50}$. The evidence is still unclear about the progression of asymptomatic osteonecrosis and small lesions; some may heal or others progress to cause significant damage to the joint ${ }^{50,51,52,53,54,55}$. There is also low- to very low-quality evidence about the effects of treatments on the progression of asymptomatic osteonecrosis ${ }^{56}$. There is very low- to low-quality evidence for a small association of most risk factors with osteonecrosis. In patients with a high suspicion of osteonecrosis owing to clinical symptoms, it is unclear whether laboratory tests such as aPL could be used to evaluate the risk of osteonecrosis ${ }^{57}$. The panel agreed that the accuracy of different imaging modalities would be similar in the general population and in patients with SLE, that radiographs are less expensive than bone scan with SPECT or MRI, and some regions may not have access to the latter.

\section{Peripartum Assessment Recommendations}

Best clinical practice (Peripartum; Supplementary Data 8, Peripartum Assessment, available with the online version of this article). Best practice dictates that all women living with SLE who are planning a pregnancy or who become pregnant should discuss their individual situations with experts in the area, with referral to an SLE care provider and obstetrical care providers, and an overall plan should be made for their pregnancy care.

Best practice dictates that for women with SLE, a complete history, physical, and laboratory evaluation should be provided immediately prior to pregnancy and each trimester of pregnancy, and when flare is suspected during pregnancy. Laboratory evaluation should include aPL (see Best Clinical Practice, General Assessments) with further testing depending on results.

Remarks: These laboratory and clinical measurements should be performed to assess whether the patients have active lupus nephritis, preeclampsia, and/or increased proteinuria due to preexisting renal disease and increased cardiac output in the setting of pregnancy. Multidisciplinary care approaches with relevant caregivers including obstetrics and gynecology, maternal-fetal medicine, and nephrologists is often necessary to facilitate the evaluation and management of these complex patients. Of note, the renal assessments cannot be interpreted in isolation of other clinical and laboratory measurements. The 14th International Congress on Antiphospholipid Antibodies Task Force provided recommendations regarding the evaluation of aPL and treatment of APS ${ }^{58}$.

Peripartum. (9) For women with SLE, we recommend that anti-Ro and anti-La antibodies be measured prior to pregnancy or during the first trimester (strong recommendation, low-quality evidence).

Remarks: Congenital heart block (CHB) occurs in only $1-2 \%$ of anti-Ro/La antibodies-positive pregnancies, but the vast majority of CHB cases arise from anti-Ro/La-positive mothers. Although screening for anti-Ro/La antibodies prior to pregnancy and/or performing fetal echocardiography in anti-Ro/La antibodies-positive pregnant women have not been shown to improve outcomes, early detection of CHB in

Personal non-commercial use only. The Journal of Rheumatology Copyright @ 2018 . All rights reserved. 
anti-Ro/La-positive women is likely to influence management. Management of positive laboratory results is beyond the scope of these recommendations.

(10) For pregnant women with SLE, we suggest that uterine and umbilical Doppler studies be performed in the second or third trimester, or at the time of a suspected flare (conditional recommendation; low-quality evidence). Timing is at the discretion of the maternal-fetal medicine or obstetrical specialist who is involved in the care (e.g., screening for placental insufficiency).

(11) For pregnant women with prior or active lupus nephritis, we suggest measuring serum creatinine and urine protein to creatinine ratio every 4-6 weeks, or more frequently if clinically indicated. We suggest blood pressure and urinalysis be measured prior to pregnancy and every 4-6 weeks until 28 weeks, every 1-2 weeks until 36 weeks, and then weekly until delivery (conditional recommendation; low-quality evidence).

Justifications (Recommendations 9-11): There is no evidence assessing the effect of providing or not providing additional screening tests on outcomes of pregnant women who have SLE. Studies show that women with SLE likely have greater adverse pregnancy outcomes than women in the general population, and a greater number of SLE flares, which may lead to greater adverse pregnancy outcomes ${ }^{59,60}$. In addition, about $1-2 \%$ of anti-Ro-exposed pregnancies in women with SLE result in babies with CHB compared to $<0.01 \%$ in the general population ${ }^{59,60}$, which the panel agreed was associated with significant management implications and morbidity in the peripartum period. There may be more SLE pregnancies with Doppler anomalies than without anomalies between 24 and 35 weeks of gestation ${ }^{59,60,61,62}$. Among pregnant women with prior or active renal disease, adverse pregnancy outcomes are greater than among those without renal disease ${ }^{59,60}$. The panel agreed that additional followup visits and testing would be acceptable, feasible, and would not increase resources and costs because of the overall small numbers of pregnant women with SLE, and would probably increase equity by ensuring standard monitoring. Management of aPL was not in the scope of these recommendations but is considered important in peripartum assessments of patients with SLE.

\section{Cervical Cancer Screening Recommendations}

Cervical cancer screening (Supplementary Data 9, available with the online version of this article). (12) For all female adult patients with SLE who are or have been sexually active, regardless of sexual orientation, we suggest annual cervical cancer screening rather than screening every 3 years at least up to the age of 69 (conditional recommendation; low-quality evidence).

Remarks: Recommendations for the general population are based on age and should also apply to women with $\mathrm{SLE}^{63}$. The Canadian general population screening interval is every
1-3 years depending on age, risk, and jurisdiction. However, women with SLE, in particular those who receive immunosuppressive medications, are recognized to be a high-risk group. The decision to stop screening after the age of 69 is individualized and based on a lifetime history of normal Pap test results.

Justification: There is no evidence in women with SLE comparing different intervals between screening for cervical cancer lesions and the effects on outcomes. There is high-quality evidence for screening every 3 years in the general population with cytology ${ }^{63}$. However, studies show that the prevalence of cervical lesions, human papilloma virus infection, and cancer are likely higher in women with SLE, and may be greater in women treated with immunosuppression $^{64,65}$. The panel agreed that providing more frequent screening would be acceptable and feasible, and result in negligible costs given current practice.

Infection Screening and Vaccination Recommendations Influenza vaccination (Supplementary Data 10, Influenza; 11, Hepatitis B; 12, Hepatitis C, available with the online version of this article). (13) We recommend that adults and children with SLE receive an annual inactivated influenza vaccination in a single dose (strong recommendation, moderate-quality evidence).

Remarks: It is important that clinicians ascertain influenza vaccination status by asking adults and children with SLE during the clinic visit. Education about the benefits of the influenza vaccination is an important part of the consultation. See also the National Advisory Committee on Immunization recommendations to avoid the use of live attenuated influenza vaccine in immunocompromised populations (www.phac-aspc.gc.ca/naci-ccni/flu-2015-grippe-eng.php).

Justification: In people with SLE, there is moderate-quality evidence that there are likely large reductions in influenza and influenza-like illness and related serious adverse events, and a trivial number of adverse outcomes and flares related to SLE with annual influenza vaccination in a single dose $66,67,68,69$. There is likely no difference in benefits when providing 1 or 2 vaccine doses. The costs to society would likely be minimally increased if the overall numbers of people vaccinated increased, and the individual and cost-effective benefits potentially large. Influenza vaccinations are acceptable to people with SLE and healthcare providers, and are funded by healthcare payers. The influenza vaccination is presently feasible to implement, and providing universal access to the vaccine would probably increase equity.

Hepatitis B screening. (14) For adult and pediatric patients with a diagnosis of SLE and high-risk behaviors for hepatitis $B$ virus (HBV) acquisition, we recommend screening for $\mathrm{HbsAg}$ and repeating according to recommendations for the general population. For patients being considered for

Personal non-commercial use only. The Journal of Rheumatology Copyright $\subset$ $\subset$ 2018. All rights reserved 
immunomodulatory therapy, we suggest screening before starting treatment (conditional recommendation, low-quality evidence).

Justification: The panel agreed that the evidence for screening in the general population with high-risk behaviors for HBV would apply to people with SLE. In addition, people with SLE receiving immunomodulatory agents and positive for HbsAg may have a high risk of $\mathrm{HBV}$ reactivation. $\mathrm{HBV}$ reactivation may increase the risk of hepatic injury and death in these patients ${ }^{70-76}$. However, prophylaxis with antiviral agents prior to or concomitantly with immunomodulatory therapy may reduce the risk of reactivation ${ }^{76}$. The applicability of these recommendations to patients with SLE who are solely taking antimalarial medication remains uncertain, given that previous studies have shown that antimalarials reduce the risk of infection in patients with $\mathrm{SLE}^{77}$.

In contrast, some studies identified cases of reactivation in patients with SLE taking hydroxychloroquine (HCQ); however, these occurred in combination with other medications ${ }^{72}$, and the likelihood of reactivation in patients taking HCQ could not be statistically determined. Reactivation of hepatitis B can also occur in patients not receiving immunomodulatory therapy ${ }^{78}$. Overall, the likelihood of reactivation in patients taking antimalarials is probably low, although this cannot be determined to a high degree of certainty based on the level of evidence. The costs of not screening and the consequences of hepatic injury and death outweigh the negligible costs of screening ${ }^{79}$. Screening is probably feasible and acceptable to both patients and healthcare providers because it is a minimally invasive test, and providing screening probably has no effect on health equity ${ }^{80}$.

Hepatitis $C$ screening. (15) For adults and pediatric patients with a diagnosis of SLE and high-risk behaviors for hepatitis $\mathrm{C}$ virus (HCV) acquisition, we recommend screening for $\mathrm{HCV}$ and repeating according to recommendations in the general population. For all other adult and pediatric patients with a diagnosis of SLE, we suggest screening for HCV and repeating according to recommendations in the general population (conditional recommendation, low-quality evidence).

Justification: The panel agreed that the evidence for screening in the general population with high-risk behaviors for HCV would apply to people with $\mathrm{SLE}^{81}$. The prevalence of $\mathrm{HCV}$ in people diagnosed with SLE ranges from $1 \%$ to $20.4 \%$ in studies; however, in many of these studies the baseline prevalence of $\mathrm{HCV}$ infection was higher than that in the general Canadian population ${ }^{82-90}$. No studies have reported the prevalence of $\mathrm{HCV}$ infection among Canadian patients with SLE. The prevalence of HCV infection in the general Canadian population is $0.7 \%$ (Government of Canada, 2016). The prevalence is likely higher in people exposed to infected bodily fluids, or who were born or living in high-prevalence regions ${ }^{81}$. Serious adverse events may occur in those who experience reactivation of hepatitis $\mathrm{C}$ in the setting of SLE, such as cirrhosis and hepatic failure ${ }^{91,92,93}$. Screening for hepatitis $\mathrm{C}$ infection can identify patients who may be candidates for highly effective direct-acting antiviral therapies to treat $\mathrm{HCV}$, and would allow the clinician in the meantime to avoid therapies for SLE that may have hepatotoxic effects and increase the potential for hepatic injury ${ }^{94}$. False positives may occur on screening for $\mathrm{HCV}$ antibody; however, in such situations, confirmatory testing with HCV RNA would typically be negative and would reveal these cases to be false positives ${ }^{95}$.

\section{DISCUSSION}

These recommendations address important nontherapeutic aspects of SLE care including clinical and comorbidity assessment over time. In using the GRADE process, systematic literature reviews were conducted and ETD tables were developed per recommendation following careful appraisal of the evidence for relevant outcomes ${ }^{96}$. Given that most of the recommendations focused on prognostic-type studies, the vast majority of studies were observational rather than randomized controlled trials. In most cases, no studies could be found directly comparing the effect of performing or not performing a particular intervention or test (e.g., disease score, infection screening) on important outcomes such as mortality, disease activity, or damage. Thus, the level of evidence used to inform the recommendations was not high, and instead indirect evidence was used. Decisions were typically made based on a comparison of the effects (and subsequent recommendations) in the general population with the SLE population. Information for patient preferences, feasibility, and economic burden was also an important aspect of the GRADE process and was used in a formalized process. Consideration of these additional domains provided an important Canadian context to these recommendations, which distinguishes them from others internationally.

Four strong recommendations were made regarding the SLE healthcare provider, CV risk assessment, peripartum assessment, and influenza vaccination. The evidence was strong recommending a rheumatologist as the primary SLE caregiver, largely based on moderate-quality evidence suggesting worse outcomes for patients with SLE without a rheumatologist or immunologist. For both $\mathrm{CV}$ risk assessment and influenza vaccination, much of the evidence was based on general population studies with low risk of bias and greater numbers of participants. Moreover, notable benefits from administering the influenza vaccine and performing CV risk assessment were also seen in the SLE population, despite lower numbers owing to the rarity of the disease. While the quality of evidence was low, the panel felt that measuring anti-Ro and anti-La antibodies was important in helping to detect $\mathrm{CHB}$ and influence subsequent management resulting in a strong recommendation rating.

Beyond the best clinical practice statements to complete a proper history and physical examination, the conditional

Personal non-commercial use only. The Journal of Rheumatology Copyright @ 2018 . All rights reserved. 
recommendations suggesting that disease activity and cumulative damage should be assessed using a formal method were felt to be very important by the panel. Greater weight was given toward the fact that most of these instruments are based on the items one elicits from a complete history and physical examination and supporting laboratory work. While there may be a perception that such tools are best reserved for SLE centers, the panel acknowledged that formalized disease scores are quite prevalent in other rheumatic diseases, where the scores are used per visit for monitoring and have other roles including drug reimbursement eligibility. Important factors including ease of administration and time burden were also incorporated into the decision. The type of disease activity was not specified largely because of the recognition that different centers have access to different tools through electronic medical records, experience, and local development of specific tools. The critical recognition was that if one takes a proper history and physical examination, a formalized approach using a validated instrument was straightforward; however, the most important point was ensuring a complete patient assessment.

The recommendations for osteoporosis and osteonecrosis assessment built on existing Canadian recommendations for the general population while acknowledging a probable greater risk for patients with SLE compared to the general population. The osteoporosis screening evidence focused on adult patients with SLE, and the panel acknowledged that future recommendations need to consider the special circumstances in pediatric bone health such as using fragility fracture rather than BMD as a measure of osteoporosis risk. Cervical cancer screening recommendations similarly looked to general population recommendations but suggested more frequent screening to address the probable higher risk among women with SLE. Future recommendations will consider the use of human papilloma vaccination in the pediatric and adult SLE populations given its important role in reducing cervical cancer risk in the general population.

Other than screening for anti-Ro and anti-La, peripartum assessment recommendations were conditional and based on low-quality evidence largely due to a lack of direct studies. The level of evidence emphasizes the need for studies providing more direct evidence to address these concerns. HBV and HCV screening were largely reflective of general population recommendations for high-risk behaviors. While recommendations for HBV and HCV screening were similar, the management of positive results would differ with prophylactic therapies for HBV and treatment for HCV. The panel agreed that registries should be developed to collect data about the outcomes of people who have SLE and HBV or $\mathrm{HCV}$, including reactivation.

Ultimately, these recommendations are meant to target the main healthcare providers for SLE, which include adult and pediatric rheumatologists and immunologists. Additionally, the recommendations may be useful for other important SLE healthcare providers including pediatricians, internal medicine, and subspecialists such as nephrologists and dermatologists. Moreover, they define an important common denominator of care assessment that should translate to the primary care practitioner and to the patient. Across all recommendations, the panel noted that evidence was lacking for particular subgroups, including for SLE patients with high versus low disease activity, and pediatric patients with SLE. This indicated important areas for future research. As research continues to grow in these areas, we will update the recommendations accordingly.

Future work is planned for knowledge translation, recognizing that recommendations are useful only if they are disseminated and resonate with those who should use them. Ideally, other priority areas including screening for other malignancies and vaccinations for other infections (e.g., varicella zoster) will be considered for future work, as well as a separate set of CRA GRADE-based recommendations for the treatment of SLE.

\section{ACKNOWLEDGMENT}

We sincerely appreciate and remember the contributions of co-authors Louise Bergeron and Jorge Medina-Rosas, who died unexpectedly prior to the completion of this manuscript. We thank Asvina Bissonauth, who participated in the early stages of recommendation development. We thank Glen Hazlewood and the CRA Guidelines Committee for review and endorsement of the paper. We also thank the CRA, the Canadian Network for Improved Outcomes in Systemic Lupus Erythematosus, and Lupus Canada for their support of these recommendations.

\section{ONLINE SUPPLEMENT}

Supplementary material accompanies the online version of this article.

\section{REFERENCES}

1. Carter EE, Barr SG, Clarke AE. The global burden of SLE: prevalence, health disparities and socioeconomic impact. Nat Rev Rheumatol 2016;12:605-20.

2. Bissonauth A, Bernatsky S, Fortin P, Gladman D, Peschken C, Urowitz M, et al. Canadian Rheumatology Association meeting Whistler, British Columbia, Canada February 24 to March 1, 2014. J Rheumatol 2014;41:1551.

3. Mosca M, Tani C, Aringer M, Bombardieri S, Boumpas D, Brey R, et al. European League Against Rheumatism recommendations for monitoring patients with systemic lupus erythematosus in clinical practice and in observational studies. Ann Rheum Dis 2010;69:1269-74.

4. Hahn BH, McMahon MA, Wilkinson A, Wallace WD, Daikh DI, Fitzgerald JD, et al. American College of Rheumatology guidelines for screening, treatment, and management of lupus nephritis. Arthritis Care Res 2012;64:797-808.

5. Gordon C, Amissah-Arthur MB, Gayed M, Brown S, Bruce IN, D'Cruz D, et al. The British Society for Rheumatology guideline for the management of systemic lupus erythematosus in adults. Rheumatology 2018;57:e1-e45.

6. Yazdany J, Panopalis P, Gillis JZ, Schmajuk G, MacLean CH, Wofsy D, et al. A quality indicator set for systemic lupus erythematosus. Arthritis Rheum 2009;61:370-7.

7. Guyatt GH, Oxman AD, Kunz R, Falck-Ytter Y, Vist GE, Liberati A, et al. Going from evidence to recommendations. BMJ 2008;336:1049-51

8. Guyatt GH, Oxman AD, Vist GE, Kunz R, Falck-Ytter Y,

Personal non-commercial use only. The Journal of Rheumatology Copyright $\subset$ $\subset$ 2018. All rights reserved 
Alonso-Coello P, et al. GRADE: an emerging consensus on rating quality of evidence and strength of recommendations. BMJ 2008;336:924-6.

9. Guyatt GH, Oxman AD, Kunz R, Jaeschke R, Helfand M, Liberati $\mathrm{A}$, et al. Incorporating considerations of resources use into grading recommendations. BMJ 2008;336:1170-3.

10. Guyatt GH, Oxman AD, Kunz R, Vist GE, Falck-Ytter Y, Schünemann HJ. What is "quality of evidence" and why is it important to clinicians? BMJ 2008;336:995-8.

11. Fiander M, McGowan J, Grad R, Pluye P, Hannes K, Labrecque M, et al. Interventions to increase the use of electronic health information by healthcare practitioners to improve clinical practice and patient outcomes. Cochrane Database Syst Rev 2015:CD004749.

12. Jaeschke R, Guyatt GH, Dellinger P, Schünemann H, Levy MM, Kunz R, et al. Use of GRADE grid to reach decisions on clinical practice guidelines when consensus is elusive. BMJ 2008;337:a744.

13. Schünemann HJ, Oxman AD, Brozek J, Glasziou P, Jaeschke R, Vist GE, et al; GRADE Working Group. Grading quality of evidence and strength of recommendations for diagnostic tests and strategies. BMJ 2008;336:1106-10.

14. Grade Working Group Website. [Internet. Accessed July 31, 2018.] Available from: www.gradeworkinggroup.org

15. Wells GA, Shea B, O'Connell D, Peterson J, Welch V, Losos M, et al. The Newcastle-Ottawa Scale (NOS) for assessing the quality of nonrandomized studies in meta-analyses. [Internet. Accessed July 31, 2018.] Available from: www.ohri.ca/programs/clinical_ epidemiology/oxford.asp

16. Hayden JA, Cote P, Bombardier C. Evaluation of the quality of prognosis studies in systematic reviews. Ann Intern Med 2006;144:427-37.

17. Higgins JP, Altman DG, Gotzsche PC, Juni P, Moher D, Oxman AD, et al. The Cochrane Collaboration's tool for assessing risk of bias in randomised trials. BMJ 2011;343:d5928.

18. Alonso-Coello P, Schunemann HJ, Moberg J, Brignardello-Petersen R, Akl EA, Davoli M, et al. GRADE Evidence to Decision (EtD) frameworks: a systematic and transparent approach to making well informed healthcare choices. 1: Introduction. BMJ 2016;353:i2016.

19. Alonso-Coello P, Oxman AD, Moberg J, Brignardello-Petersen R, Akl EA, Davoli M, et al. GRADE Evidence to Decision (EtD) frameworks: a systematic and transparent approach to making well informed healthcare choices. 2: Clinical practice guidelines. BMJ 2016;353:i2089.

20. Guyatt GH, Schünemann HJ, Djulbegovic B, Akl EA. Guideline panels should not GRADE good practice statements. J Clin Epidemiol 2015;68:597-600.

21. Andrews J, Guyatt G, Oxman AD, Alderson P, Dahm P, Falck-Ytter Y, et al. GRADE guidelines: 14. Going from evidence to recommendations: the significance and presentation of recommendations. J Clin Epidemiol 2013;66:719-25.

22. Blaauw AA, Schuwirth LW, van der Vleuten CP, Smits F, van der Linden S. Assessing clinical competence: recognition of case descriptions of rheumatic diseases by general practitioners. Br J Rheumatol 1995;34:375-9.

23. Gamez-Nava JI, Gonzalez-Lopez L, Davis P, Suarez-Almazor ME. Referral and diagnosis of common rheumatic diseases by primary care physicians. Br J Rheumatol 1998;37:1215-9.

24. Narain S, Richards HB, Satoh M, Sarmiento M, Davidson R, Shuster J, et al. Diagnostic accuracy for lupus and other systemic autoimmune diseases in the community setting. Arch Intern Med 2017;164:2435-41.

25. Ward MM. Association between physician volume and in-hospital mortality in patients with systemic lupus erythematosus. Arthritis Rheum 2005;52:1646-54

26. Ward MM. Hospital experience and mortality in patients with systemic lupus erythematosus. Arthritis Rheum 1999;42:891-8.

27. Rzany B, Coresh J, Whelton PK, Petri M. Risk factors for hypercreatinemia in patients with systemic lupus erythematosus. Lupus 1999;8:532-40.

28. Rovisco I. Hospital admission of systemic lupus erythematosus patients before and after establishment of a lupus clinic: a 10-year retrospective study (abstract). Lupus 2011;20:383.

29. Bombardier C, Gladman DD, Urowitz MB, Caron D, Chang CH. Derivation of the SLEDAI. A disease activity index for lupus patients. The Committee on Prognosis Studies in SLE. Arthritis Rheum 1992;35:630-40.

30. Symmons DP, Coppock JS, Bacon PA, Bresnihan B, Isenberg DA, Maddison P, et al. Development and assessment of a computerized index of clinical disease activity in systemic lupus erythematosus. Members of the British Isles Lupus Assessment Group (BILAG). Q J Med 1988;69:927-37.

31. Fellows of Harvard College. SLE Activity Measure-Revised (SLAM-R) 1998

32. Keeling S, Vandermeer B, Medina J, Chatterley T, Nevskaya T, Pope J, et al. Measuring disease activity and damage with validated metrics: a systematic review on mortality and damage in systemic lupus erythematosus. J Rheumatol 2018;45:1448-61

33. Gladman D, Ginzler E, Goldsmith C, Fortin P, Liang M, Urowitz M, et al. The development and initial validation of the Systemic Lupus International Collaborating Clinics/American College of Rheumatology damage index for systemic lupus erythematosus. Arthritis Rheum 1996;39:363-9.

34. Tselios K, Sheane BJ, Gladman DD, Urowitz MB. Optimal monitoring for coronary heart disease risk in patients with systemic lupus erythematosus: a systematic review. J Rheumatol 2016; 43:54-65.

35. Anderson TJ, Grégoire J, Pearson GJ, Barry AR, Couture P, Dawes M, et al. 2016 Canadian Cardiovascular Society Guidelines for the Management of Dyslipidemia for the Prevention of Cardiovascular Disease in the Adult. Can J Cardiol 2016;32:1263-82.

36. Canadian Diabetes Association Clinical Practice Guidelines Expert Committee, Booth G, Cheng AY. Canadian Diabetes Association 2013 clinical practice guidelines for the prevention and management of diabetes in Canada. Methods. Can J Diabetes 2013;37 Suppl 1:4

37. Kanis JA, Johnell O, Oden A, Johansson H, McCloskey E. FRAX and the assessment of fracture probability in men and women from the UK. Osteoporos Int 2008;19:385-97.

38. Papaioannou A. 2010 clinical practice guidelines for the diagnosis and management of osteoporosis in Canada: summary. CMAJ 2010;182:1864-73

39. Mendoza-Pinto C, Garcia-Carrasco M, Sandoval-Cruz H, Munoz-Guarneros M, Escarcega RO, Jimenez-Hernandez M, et al. Risk factors of vertebral fractures in women with systemic lupus erythematosus. Clin Rheumatol 2009;28:579-85.

40. Ramsey-Goldman R, Dunn JE, Huang CF, Dunlop D, Rairie JE, Fitzgerald $\mathrm{S}$, et al. Frequency of fractures in women with systemic lupus erythematosus: comparison with United States population data. Arthritis Rheum 1999;42:882-90

41. Boyanov M, Robeva R, Popivanov P. Bone mineral density changes in women with systemic lupus erythematosus. Clin Rheumatol 2003;22:318-23

42. Almehed K, Forsblad d'Elia H, Kvist G, Ohlsson C, Carlsten H. Prevalence and risk factors of osteoporosis in female SLE patients-extended report. Rheumatology 2007;46:1185-90

43. Mendoza-Pinto C, Garcia-Carrasco M, Sandoval-Cruz H, Escarcega RO, Jimenez-Hernandez M, Etchegaray-Morales I, et al. Risks factors for low bone mineral density in pre-menopausal Mexican women with systemic lupus erythematosus. Clin Rheumatol 2009;28:65-70

44. Pineau CA, Urowitz MB, Fortin PJ, Ibanez D, Gladman DD.

Personal non-commercial use only. The Journal of Rheumatology Copyright (C) 2018. All rights reserved. 
Osteoporosis in systemic lupus erythematosus: factors associated with referral for bone mineral density studies, prevalence of osteoporosis and factors associated with reduced bone density. Lupus 2004;13:436-41.

45. Borba VZ, Vieira JG, Kasamatsu T, Radominski SC, Sato EI, Lazaretti-Castro M. Vitamin D deficiency in patients with active systemic lupus erythematosus. Osteoporos Int 2009;20:427-33.

46. Kamen DL, Cooper GS, Bouali H, Shaftman SR, Hollis BW, Gilkeson GS. Vitamin D deficiency in systemic lupus erythematosus. Autoimmun Rev 2006;5:114-7.

47. Toloza S, Cole D, Gladman D, Ibañez D, Urowitz M. Vitamin D insufficiency in a large female SLE cohort. Lupus 2010;19:13-9.

48. Bultink IE, Lems WF, Kostense PJ, Dijkmans BA, Voskuyl AE. Prevalence of and risk factors for low bone mineral density and vertebral fractures in patients with systemic lupus erythematosus. Arthritis Rheum 2005;52:2044-50

49. Mont MA, Glueck CJ, Pacheco IH, Wang P, Hungerford DS, Petri M. Risk factors for osteonecrosis in systemic lupus erythematosus. J Rheumatol 1997;24:654-62.

50. Oinuma K, Harada Y, Nawata Y, Takabayashi K, Abe I, Kamikawa $\mathrm{K}$, et al. Osteonecrosis in patients with systemic lupus erythematosus develops very early after starting high dose corticosteroid treatment. Ann Rheum Dis 2001;60:1145-8.

51. Ehmke TA, Cherian JJ, Wu ES, Jauregui JJ, Banerjee S, Mont MA. Treatment of osteonecrosis in systemic lupus erythematosus: a review. Curr Rheumatol Rep 2014;16:8.

52. Mont MA, Baumgarten KM, Rifai A, Bluemke DA, Jones LC, Hungerford DS. Atraumatic osteonecrosis of the knee. J Bone Joint Surg Am 2000;82:1279-90.

53. Nakamura J, Harada Y, Oinuma K, Iida S, Kishida S, Takahashi K. Spontaneous repair of asymptomatic osteonecrosis associated with corticosteroid therapy in systemic lupus erythematosus: 10-year minimum follow-up with MRI. Lupus 2010;19:1307-14.

54. Mont MA, Zywiel MG, Marker DR, McGrath MS, Delanois RE. The natural history of untreated asymptomatic osteonecrosis of the femoral head: a systematic literature review. J Bone Joint Surg Am 2010;92:2165-70.

55. Aranow C, Zelicof S, Leslie D, Solomon S, Barland P, Norman A, et al. Clinically occult avascular necrosis of the hip in systemic lupus erythematosus. J Rheumatol 1997;24:2318-22.

56. Hungerford DS, Jones LC. Asymptomatic osteonecrosis: should it be treated? Clin Orthop Relat Res 2004;429:124-30.

57. Hussein S, Suitner M, Béland-Bonenfant S, Baril-Dionne A, Vandermeer B, Santesso N, et al. Monitoring of osteonecrosis in systemic lupus erythematosus: a systematic review and metaanalysis. J Rheumatol 2018;45:1462-76.

58. Erkan D, Aguiar CL, Andrade D, Cohen H, Cuadrado MJ, Danowski A, et al. 14th International Congress on Antiphospholipid Antibodies Task Force report on antiphospholipid syndrome treatment trends. Autoimmun Rev 2014;13:685-96.

59. Buyon JP, Kim MY, Guerra MM, Laskin CA, Petri M, Lockshin MD, et al. Predictors of pregnancy outcomes in patients with lupus: a cohort study. Ann Intern Med 2015;163:153-63.

60. McDonald EG, Bissonette L, Ensworth S, Dayan N, Clarke AE, Keeling S, et al. Monitoring of systemic lupus erythematosus pregnancies: a systematic literature review. J Rheumatol 2018;45:1477-90.

61. Le Thi Huong D, Wechsler B, Vauthier-Brouzes D, Duhaut P, Costedoat N, Andreu MR, et al. The second trimester Doppler ultrasound examination is the best predictor of late pregnancy outcome in systemic lupus erythematosus and/or the antiphospholipid syndrome. Rheumatol 2006;45:332-8.

62. Farine D, Granovsky-Grisaru S, Ryan G, Seaward PG, Teoh TG, Laskin C, et al. Umbilical artery blood flow velocity in pregnancies complicated by systemic lupus erythematosus. J Clin Ultrasound
1998;26:379-82.

63. Dickinson J, Tsakonas E, Conner Gorber S, Lewin G, Shaw E, et al Canadian Task Force on Preventive Health Care. Recommendations on screening for cervical cancer. CMAJ 2013;185:35-45.

64. Zard E, Arnaud L, Mathian A, Chakhtoura Z, Hie M, Touraine P, et al. Increased risk of high grade cervical squamous intraepithelial lesions in systemic lupus erythematosus: a meta-analysis of the literature. Autoimmun Rev 2014;13:730.

65. Dugué P, Lynge E, Rebolj M. Increased risk of high-grade squamous intraepithelial lesions in systemic lupus erythematosus: additional data from Denmark. Autoimmun Rev 2014;13:1241-2.

66. Aikawa N, Trudes G, Campos L, Pereira R, Moraes J, Ribeiro A, et al. Immunogenicity and safety of two doses of a non-adjuvanted influenza A H1N1/2009 vaccine in young autoimmune rheumatic diseases patients. Lupus 2013;22:1394-8.

67. Aikawa NE, Campos LM, Silva CA, Carvalho JF, Saad CG, Trudes $\mathrm{G}$, et al. Glucocorticoid: major factor for reduced immunogenicity of 2009 influenza A (H1N1) vaccine in patients with juvenile autoimmune rheumatic disease. J Rheumatol 2012;39:167-73.

68. Beck CR, McKenzie BC, Hashim AB, Harris RC, Zanuzdana A, Agboado G, et al. Influenza vaccination for immunocompromised patients: systematic review and meta-analysis from a public health policy perspective. PLoS One 2011;6:e29249.

69. Beck CR, McKenzie BC, Hashim AB, Harris RC, Nguyen-Van-Tam JS. Influenza vaccination for immunocompromised patients: systematic review and meta-analysis by etiology. J Infect Dis 2012;206:1250-9.

70. Yang S, Lin WT, Chen DY, Chang CS, Yeh HZ. Oral antiviral prophylaxis is effective in preventing HBV reactivation in HBsAg-positive systemic lupus erythematosus patients undergoing immunosuppressive therapy [abstract]. Hepatology 2013;58 Suppl 1:907.

71. Tse KC, Yung S, Tang C, Yip TP, Chan TM. Management of hepatitis $\mathrm{B}$ reactivation in patients with lupus nephritis. Rheumatol Int 2009;29:1273-7.

72. Thong BY, Koh ET, Chng HH, Chow WC. Outcomes of chronic hepatitis B infection in Oriental patients with rheumatic diseases. Ann Acad Med Singapore 2007;36:100-5

73. Laohapand C, Arromdee E, Tanwandee T. Long-term use of methotrexate does not result in hepatitis B reactivation in rheumatologic patients. Hepatol Int 2015;9:202-8.

74. Kato M, Atsumi T, Kurita T, Odani T, Fujieda Y, Otomo K, et al. Hepatitis B virus reactivation by immunosuppressive therapy in patients with autoimmune diseases: risk analysis in Hepatitis B surface antigen-negative cases. J Rheumatol 2011;38:2209-14.

75. Cheng J, Li JB, Sun QL, Li X. Reactivation of hepatitis B virus after steroid treatment in rheumatic diseases. J Rheumatol 2011;38:181-2.

76. Kalyoncu U, Yonem O, Calguneri M, Ersoy O, Karadag O, Akdogan A, et al. Prophylactic use of lamivudine with chronic immunosuppressive therapy for rheumatologic disorders. Rheumatol Int 2009;29:777-80.

77. Ruiz-Irastorza G, Olivares N, Ruiz-Arruza I, Martinez-Berriotxoa A, Egurbide E, Aguirre C. Predictors of major infections in systemic lupus erythematosus. Arthritis Res Ther 2009;11:R109.

78. Kamitsukasa H, Iri M, Tanaka A, Nagashima S, Takahashi M, Nishizawa T, et al. Spontaneous reactivation of hepatitis B virus (HBV) infection in patients with resolved or occult HBV infection. J Med Virol 2015;87:589-600

79. He J, Bowen J, Xie F, Goeree R. Cost-effectiveness analysis of antiviral treatments for $\mathrm{HBeAg}$-positive chronic hepatitis B in Canada. Value Health 2012;15:894-906.

80. Lim SG, Aung MO, Chung SW, Soon CS, Mak BH, Lee KH. Patient preferences for hepatitis B therapy. Antivir Ther 2013;18:663-70

81. AASLD/IDSA HCV Guidance Panel. Hepatitis C guidance:

Personal non-commercial use only. The Journal of Rheumatology Copyright (C) 2018. All rights reserved 
AASLD-IDSA recommendations for testing, managing, and treating adults infected with hepatitis C virus. Hepatology 2015;62:932-54.

82. Barbosa VS, Silva NA, Martins RM. Hepatitis C virus seroprevalence and genotypes in patients with diffuse connective tissue diseases and spondyloarthropathies. Braz J Med Biol Res 2005;38:801-5.

83. Garf El A, Zorkany El B, Gheith R, Sheba H, Abdel Moneim G, Garf El K. Prevalence and clinical presentations of hepatitis C virus among patients admitted to the rheumatology ward. Rheumatol Int 2012;32:2691-5.

84. Mohan P, Rajasekaran M, Ramanathan P, Venkataraman J. Prevalence of hepatitis $C$ virus antibodies in systemic lupus erythematosus. Eur J Gastroenterol Hepatol 2009;21:1437-8.

85. Abu-Shakra M, El-Sana S, Margalith M, Sikuler E, Neumann L, Buskila D. Hepatitis B and C viruses serology in patients with SLE. Lupus 1997;6:543-4.

86. Agmon-Levin N, Zafrir Y, Paz Z, Shilton T, Zandman-Goddard G, Shoenfeld Y. Ten cases of systemic lupus erythematosus related to hepatitis B vaccine. Lupus 2009;18:1192-7.

87. Abou-Raya A. Prevalence of antibodies against hepatitis $C$ virus in patients with systemic lupus erythematosus (abstract). Lupus 2010;19:126-7.

88. El Garf A, Shaheen N, Gaber W, Sobhy N. Prevalence and impact of chronic hepatitis $\mathrm{C}$ virus infection on the clinical manifestations and disease activity among patients suffering from systemic lupus erythematosus. Egypt Rheumatologist 2013;35:9-14.

89. Karakoç Y, Dilek K, Güllülü M, Yavuz M, Ersoy A, Akalyn H, et al. Prevalence of hepatitis $\mathrm{C}$ virus antibody in patients with systemic lupus erythematosus. Ann Rheum Dis 1997;56:570-1.
90. Ahmed MM, Berney SM, Wolf RE, Hearth-Holmes M, Hayat S, Mubashir E, et al. Prevalence of active hepatitis $\mathrm{C}$ virus infection in patients with systemic lupus erythematosus. Am J Med Sci 2006;331:252-6.

91. Mitwalli AH, Hayat A, Alwakeel J, Hammad D. Effects of concomitant hepatitis $\mathrm{C}$ virus infection in patients with underlying lupus nephritis on long-term renal outcome. Nephrol Dial Transplant 2012;27:627-32.

92. Perlemuter G, Cacoub P, Sbai A, Hausfater P, Thibault V, Le TH, et al. Hepatitis $\mathrm{C}$ virus infection in systemic lupus erythematosus: a case-control study. J Rheumatol 2003;30:1473-8.

93. Ramos-Casals M, Jara LJ, Medina F, Rosas J, Calvo-Alen J, Mañá J, et al. Systemic autoimmune diseases co-existing with chronic hepatitis C virus infection (the HISPAMEC Registry): patterns of clinical and immunological expression in 180 cases. J Intern Med 2005;257:549-57.

94. Jazwinski AB, Muir AJ. Direct-acting antiviral medications for chronic hepatitis $\mathrm{C}$ virus infection. Gastroenterol Hepatol 2011;7:154-62.

95. Kowdley KV, Subler DE, Scheffel J, Moore B, Smith H. Hepatitis C virus antibodies in systemic lupus erythematosus. J Clin Gastroenterol 1997;25:437-9.

96. Schünemann HJ, Mustafa R, Brozek J, Santesso N, Alonso-Coello P, Guyatt G, et al. GRADE Guidelines: 16. GRADE evidence to decision frameworks for tests in clinical practice and public health. J Clin Epidemiol 2016;76:89-98. 